\title{
Design of photonic crystal waveguides for evanescent coupling to optical fiber tapers and integration with high- $Q$ cavities
}

\author{
Paul E. Barclay, Kartik Srinivasan, and Oskar Painter \\ Department of Applied Physics, California Institute of Technology, Pasadena, California 91125
}

Received November 15, 2002; revised manuscript received July 2, 2003; accepted July 31, 2003

\begin{abstract}
We describe a novel scheme based on evanescent guided-wave coupling for optically interfacing between conventional fiber-optic and planar photonic crystal devices such as waveguides and resonant cavities. By considering the band structure of bulk photonic crystal slabs, we analyze the $\mathbf{k}$ space properties of a linear defect waveguide and establish a set of design rules to ensure efficient evanescent coupling with optical fiber tapers. These rules are used to design a waveguide in a square-lattice photonic crystal. The coupling efficiency is calculated with a coupled-mode theory incorporating the finite-difference time-domain-calculated uncoupled modes of the fiber taper and photonic crystal waveguide. On the basis of this coupled-mode theory, $95 \%$ power transfer from the fiber taper to the photonic crystal waveguide is possible over a coupling length of 80 lattice periods and with a bandwidth of $1.5 \%$ of the center wavelength. The integration of this waveguide with a photonic crystal defect resonant cavity is also presented, thus showing the usefulness of the combined fiber taper and photonic crystal waveguide system for efficient, optical fiber-based probing of optical elements based on planar photonic crystal technologies. (C) 2003 Optical Society of America
\end{abstract}

OCIS codes: $130.2790,230.7370$.

\section{INTRODUCTION}

Since the first proposal of photonic bandgap (PBG) materials and their use for spontaneous emission control, ${ }^{1}$ high-index-contrast periodic dielectric structures, photonic crystals (PCs), have promised novel control of the propagation and dispersion of light. Two-dimensional (2-D) PC slab waveguide structures ${ }^{2-5}$ offer a practical geometry for the implementation of PC devices by means of conventional planar lithography and semiconductor processing techniques. Planar PC technologies in a variety of material systems, such as silicon-on-insulator, GaAs, and InP, have been used to realize such devices as waveguides, ${ }^{6,7}$ lasers, ${ }^{8}$ coupled-resonator waveguide systems, ${ }^{9-11}$ and add-drop filters, ${ }^{12,13}$ to name just a few. Resonant cavities formed in planar PC slabs, with mode volumes approaching a cubic half-wavelength, have also attracted some interest for use in cavity QED experiments $^{14-16}$ and in single-photon sources. ${ }^{17-19}$ The utility of these types of device relies heavily on one's ability to efficiently couple light into and out of the planar PC.

Because of the large refractive index of current planar PC-based materials (required for high-index-contrast structures), the spatial mismatch between optical fiber modes, or diffraction-limited free-space beams, and single-mode PC devices is severe. Recent progress in the design of adiabatic and tapered transitions ${ }^{20-22}$ between standard index-guided waveguides and PC waveguides has improved the theoretical efficiency of end-fire coupling schemes. However, because many of the applications and advantages of planar PCs are seen in the integration and miniaturization of components, the long adiabatic transitions from high-index index-guided waveguides with relatively small mode sizes to weakly guided fiber-sized modes that are required to interface with fiber optics ${ }^{23}$ are not desirable. Out-of-plane coupling schemes have also been studied, ${ }^{24}$ but they currently suffer from high loss and a relatively high complexity. Evanescent coupling between a fiber taper ${ }^{25}$ and a PC waveguide, as detailed schematically in Fig. 1, buries much of the complexity of on-chip coupling within the fiber and makes use of the inherent dispersive properties of PCs to enable efficient guided-wave coupling to silica optical fibers. A single fiber taper used in this manner can function as an adjustable wafer-scale probe for the testing of multiple devices on a planar chip. This evanescent coupling technique is also suitable for adaptation to planar lightwave circuit technology, allowing for the interfacing between fiber optics and PCs in a mechanically robust and scalable fashion.

Recently, a similar evanescent coupling scheme was independently proposed in Ref. 26, in which a numerical analysis was presented with a parallelized finitedifference time-domain (FDTD) solver. In this paper we design a PC waveguide for efficient fiber taper coupling using an approximate coupled-mode theory in combination with FDTD simulations. This analysis allows us to determine, in a general manner, the important PC waveguide properties for efficient, mode-selective, evanescent coupling to optical fiber tapers or other similar low-index waveguides such as those used in silica-on-silicon planar lightwave circuits. Note that since this paper's original submission this coupling scheme has been demonstrated experimentally in Ref. 27.

In the simplest picture, evanescent coupling between two parallel waveguides requires that there exist (in the frequency range of interest) a pair of modes, one from 
(a)

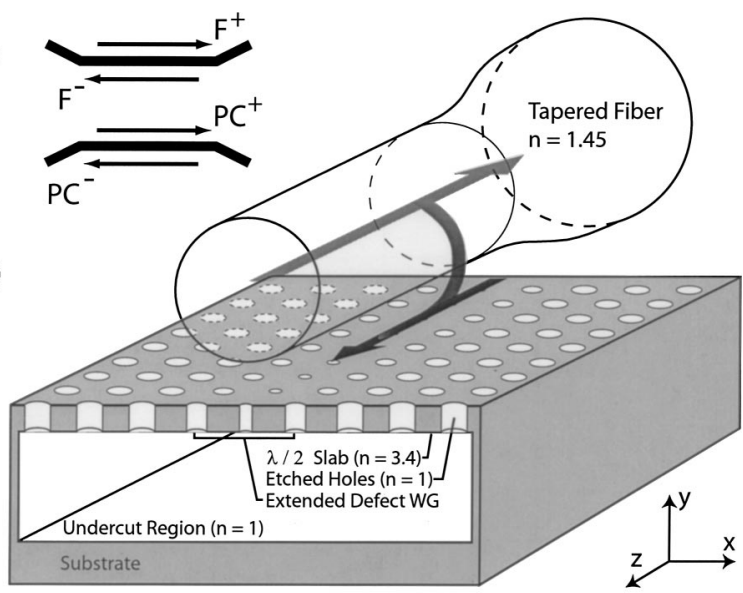

(c)

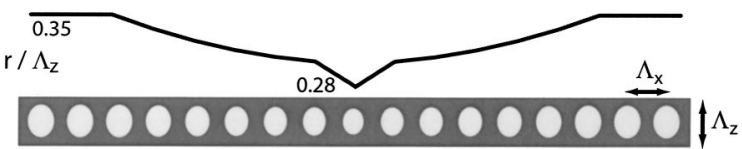

Fig. 1. (a) Schematic of the coupling scheme showing the fourmode basis used in the coupled-mode theory. (b) Coupling geometry. In the case considered here the coupling is contradirectional. WG, waveguide. (c) Grading of the hole radius used to form the waveguide and a top view of the graded-defect compressed-lattice $\left(\Lambda_{x} / \Lambda_{z}=0.8\right)$ waveguide unit cell.

each waveguide, that share a common momentum component down the waveguide (phase matching) and for which their transverse profiles and electric field polarizations are similar. A weak spatial overlap of the evanescent tails of each mode can then result in significant power transfer between the waveguides. Full power transfer requires that, in addition, no other radiation or guided modes of either waveguide participate in the coupling, either due to a large phase mismatch or weak transverse overlap. Fiber taper coupling has been shown to be extremely valuable in this regard (compared with simple prism coupling that involves a continuum of modes), providing near-perfect single-mode to single-mode coupling between tapers and dielectric microsphere ${ }^{28}$ and toroid ${ }^{29}$ resonators for ultrasensitive measurement of high- $Q$ whispering-gallery modes. In a similar manner fiber taper probes can be used to couple to 2-D PC membrane waveguides thanks to their undercut air-bridge structure that suppresses radiation from the fiber into the substrate and their zone-folded dispersion that enables phase matching between the dissimilar fiber and the PC modes. Thus, by designing a PC waveguide whose defect mode has a transverse field profile that sufficiently overlaps the fiber taper's fundamental mode field profile, we can achieve efficient power transfer between the waveguides. Furthermore, the flexibility in lattice engineering afforded by PCs allows this waveguide to simultaneously be designed to couple efficiently to PC defect cavities, providing a fiber-PC waveguide-PC cavity optical probe. In this paper we discuss the design of a PC waveguide that satisfies the requirements outlined above. In Section 2 a simple coupled-mode theory that models coupling between a fiber taper and a PC is presented, and the desired waveguide properties are illuminated mathematically and discussed in more detail. In Section 3 a general $\mathbf{k}$ space analysis of bulk PC band structures is used to de- termine which types of defect mode have the desired properties. The results from these sections are then applied to the design and analysis of a PC waveguide in a square lattice in Section 4 and are further illustrated with FDTD supermode calculations in Section 5. Finally in Section 6 we show how this waveguide can be used to probe high- $Q$ $\mathrm{PC}$ resonant cavities.

\section{COUPLED-MODE THEORY}

It has long been realized that modes in translationally invariant waveguides with differing dielectric constants can be phase matched with the aid of a grating, so it is not surprising that the intrinsic discrete translational symmetry of PC waveguides and the resulting zone-folded dispersion of their modes allows PCs to be phase matched with a large class of dissimilar waveguides including tapered fibers. Grating-mediated phase-matching schemes have been studied extensively beginning with the research of microwave traveling-wave tubes ${ }^{30}$ and more recently of optical devices such as filters, directional couplers, and distributed feedback lasers (Ref. 31 and references therein). However, because the dielectric contrast of a PC grating is large, fiber-PC coupling differs from that of a traditional (weak) grating-assisted coupler; rather than analyze coupling between plane waves of the untextured waveguides, the Bloch eigenmodes of the PC must be considered. Although rigorous coupled-mode theories for Bloch modes have been developed in the context of nonlinear perturbations to Bragg fibers, ${ }^{32}$ photonic crystals, ${ }^{33}$ and coupled-resonator optical waveguides, ${ }^{34}$ none of these formalisms consider coupling between parallel waveguides. To evaluate the properties of evanescent coupling between a fiber and a PC defect waveguide, we present a coupled-mode theory that allows us to approximately predict the power transfer between the PC Bloch modes and the fiber plane-wave modes as a function of propagation distance, transverse coupling strength, and phase mismatch.

The physical system that we model is specified by the dielectric constants of the interacting waveguides $\epsilon_{\mu}(\mathbf{r})$, each of which individually supports a set of modes $\mathbf{E}_{\nu}{ }^{\mu}(\mathbf{r})$, where $\mu$ labels the waveguides and $\nu$ labels the eigenmodes of each waveguide. For $\exp (-i \omega t)$ time dependence, Maxwell's equations require that each of these modes satisfies the eigenvalue equation

$$
\nabla \times \nabla \times \mathbf{E}_{\nu}{ }^{\mu}(\mathbf{r})=\widetilde{\omega}^{2} \epsilon_{\mu}(\mathbf{r}) \mathbf{E}_{\nu}{ }^{\mu}(\mathbf{r}),
$$

where $\widetilde{\omega}=\omega / c$ is the free-space wave number.

The fundamental approximation of waveguide coupledmode theories is that, after some propagation distance, the field of the composite system represented by $\epsilon(\mathbf{r})$ $=\epsilon_{1} \cap \epsilon_{2} \ldots \cap \epsilon_{n}$ can be approximated by some linear combination of the modes of the constituent systems represented by $\epsilon_{\mu}(\mathbf{r})$ :

$$
\mathbf{E}(\mathbf{r})=\sum_{\mu \nu} C_{\nu}{ }^{\mu}(z) \mathbf{E}_{\nu}{ }^{\mu}(\mathbf{r}),
$$

where we assumed that the modes are propagating in the $\pm \hat{z}$ direction, or more precisely that the power flux of the individual modes in the $\hat{z}$ direction is constant. When we 
consider continuums of delocalized modes, an integral replaces the discrete sum. If $\epsilon_{\mu}(\mathbf{r})$ is periodic in $z$ so that $\epsilon_{\mu}\left(x, y, z+\Lambda_{z}\right)=\epsilon_{\mu}(x, y, z)$, by Bloch's theorem ${ }^{35}$ the eigenmodes have $z$ dependence of the form $\mathbf{E}_{\nu}{ }^{\mu}(x, y$, $\left.z+\Lambda_{z}\right)=\exp \left(i \beta_{\nu} \Lambda_{z}\right) \mathbf{E}_{\nu}{ }^{\mu}(x, y, z)$ and Eq. (1) becomes

$$
\mathbb{H}_{\beta_{\nu}} \mathbf{e}_{\beta_{\nu}}{ }^{\mu}(\mathbf{r})=\widetilde{\omega}^{2} \epsilon_{\mu}(\mathbf{r}) \mathbf{e}_{\beta_{\nu}}{ }^{\mu}(\mathbf{r}),
$$

where

$$
\mathbb{H}_{\beta_{\nu}}=\left[-\beta_{\nu}{ }^{2} \hat{z} \times \hat{z}+i \beta_{\nu}(\hat{z} \times \nabla+\nabla \times \hat{z})+\nabla \times \nabla\right] \times,
$$

$\mathbf{e}_{\beta_{\nu}}{ }^{\mu}\left(x, y, z+\Lambda_{z}\right)=\mathbf{e}_{\beta_{\nu}}{ }^{\mu}(x, y, z), \quad$ and $\quad-\pi / \Lambda_{z}<\beta_{\nu}$ $\leqslant \pi / \Lambda_{z}$ (i.e., $\beta_{\nu}$ is restricted to the first Brillouin zone) so that the eigenmodes are not overcounted. Equation (3) is often solved as an eigenvalue problem for $\widetilde{\omega}_{\nu}$ parameterized by the wave number $\beta$, giving a dispersion relation $\widetilde{\omega}_{\nu}=\widetilde{\omega}_{\nu}(\beta)$. In linear media, only modes degenerate in $\widetilde{\omega}$ have nonzero time-averaged coupling over typical laboratory time scales, and it is convenient to label the modes at fixed $\widetilde{\omega}$ by their wave number $\beta_{\nu}(\widetilde{\omega})$. Both conventions are equivalent and interchangeable. Typically (as discussed below) for weak coupling, only modes nearly resonant in $\beta$ (modulo a reciprocal lattice vector $2 \pi / \Lambda_{z}$ ) to the exciting field need to be included in Eq. (2); this is the basic assumption of the coupled-mode theory. For weak coupling this assumption that only nearly resonant modes interact is reasonable; however, the question of completeness is less clear. In general Eq. (2) cannot satisfy Maxwell's equations because the eigenmodes of waveguide $\mu_{1}$ do not satisfy the boundary conditions of waveguide $\mu_{2}$ and vice versa. This issue was debated vigorously in the late 1980s but was not resolved, it is well summarized in Ref. 36. In Ref. 37 Haus and Snyder showed that in some cases the ansatz Eq. (2) can be improved when the modes used in the expansion are modified so that they satisfy the boundary conditions of the composite system. This improvement is nontrivial in the case of a PC slab, however, and is not used here. This deficiency is minimized for TE-like modes but exists nonetheless if the waveguides lack translational invariance or planar geometry as is the case in PC waveguides and fiber tapers, respectively. Despite this limitation we proceed under the assumption that, in the limit of weak coupling, the resulting model is a useful design tool that correctly describes the dependence of the coupling on the physical parameters but whose absolute results may deviate from the exact values.

To formulate coupled-mode equations we assume that ansatz Eq. (2) is a solution to Maxwell's equations for the hybrid system and we employ the Lorentz reciprocity relationship $^{38}$ that must hold for any two solutions to Maxwell's equations in nonmagnetic materials:

$$
\begin{aligned}
& \frac{\partial}{\partial z} \int_{z}\left(\mathbf{E}_{1} \times \mathbf{H}_{2} *+\mathbf{E}_{2} *\right.\left.\times \mathbf{H}_{1}\right) \cdot \hat{z} \mathrm{~d} x \mathrm{~d} y \\
&=i \widetilde{\omega} \int_{z} \mathbf{E}_{1} \cdot \mathbf{E}_{2} *\left(\epsilon_{1}-\epsilon_{2}^{*}\right) \mathrm{d} x \mathrm{~d} y,
\end{aligned}
$$

where $(\mathbf{E}, \mathbf{H})_{1,2}$ satisfy Maxwell's equations for $\epsilon_{1,2}$. Setting

$$
\begin{aligned}
& \mathbf{E}_{1}=\sum_{j} C_{j}(z) \mathbf{E}_{j}(\mathbf{r}), \\
& \mathbf{E}_{2}=\mathbf{E}_{i},
\end{aligned}
$$

and correspondingly

$$
\begin{aligned}
& \epsilon_{1}=\epsilon, \\
& \epsilon_{2}=\epsilon_{i},
\end{aligned}
$$

where the single index $i=\left(\mu_{i}, \nu_{i}\right)$, labeling both the waveguide and the mode is adopted for clarity, and substituting Eqs. (6) and (7) into Eq. (5) we obtain the following power-conserving coupled-mode equations:

$$
P_{i j} \frac{\mathrm{d} C_{j}}{\mathrm{~d} z}=i \widetilde{\omega} K_{i j} C_{j},
$$

where

$$
\begin{aligned}
& P_{i j}(z)=\int_{z}\left(\mathbf{E}_{i}^{*} \times \mathbf{H}_{j}+\mathbf{E}_{j} \times \mathbf{H}_{i}^{*}\right) \cdot \hat{z} \mathrm{~d} x \mathrm{~d} y, \\
& K_{i j}(z)=\int_{z} \mathbf{E}_{i}^{*} \cdot \mathbf{E}_{j}\left(\epsilon-\boldsymbol{\epsilon}_{j}\right) \mathrm{d} x \mathrm{~d} y,
\end{aligned}
$$

and it was assumed that all dielectric constants are real. Equation (8) is similar to the coupled-mode equations given in Ref. 39, with the only differences arising from the fact that no specific form of $z$ dependence of the eigenmodes was assumed. When the mode amplitudes are fixed at some $z=z_{0}$, Eq. (8) can easily be solved numerically, giving a transfer matrix that maps the amplitudes at $z_{0}$ to $z_{0}+L$. To correctly model an experimental setup, the amplitude of the modes propagating in the $+\hat{z}$ direction should be fixed at $z_{0}$, and the amplitude of the modes propagating in the $-\hat{z}$ direction should be fixed at $z_{0}+L$. Equation (8) can be solved with these mixed boundary conditions by first calculating the transfer matrix [which maps $C_{i}{ }^{ \pm}\left(z_{0}\right) \rightarrow C_{i}{ }^{ \pm}\left(z_{0}+L\right)$ where the superscript represents the propagating direction of mode $j$ ] and then transforming it to the appropriate scattering matrix [which maps $C_{i}{ }^{+}\left(z_{0}\right) \rightarrow C_{i}{ }^{+}\left(z_{0}+L\right)$ and $C_{i}{ }^{-}\left(z_{0}\right.$ $\left.+L) \rightarrow C_{i}{ }^{-}\left(z_{0}\right)\right]$. Alternately, we can solve Eq. (8) directly using a boundary-value problem algorithm.

From Eq. (5) the diagonal terms $P_{i i}$ of the power matrix are constant and are typically normalized to plus or minus unity depending on the sign of the group velocity of mode $i$. In addition, it can be shown that Bloch modes of the same waveguide are power orthogonal so that $P_{i j}=0$ if $\epsilon_{i}=\epsilon_{j}$ and $\mathbf{E}_{i} \neq \mathbf{E}_{j}$. However, modes from neighboring waveguides are not power orthogonal, resulting in nonzero off-diagonal $z$-dependent components in $P_{i j}$ that must be retained for Eq. (8) to be power conserving. In the fiber-PC system, the $z$ dependence of the PC fields is the product of a plane-wave part and a periodic part whereas fiber fields have a plane-wave-like $z$ dependence. Expanding the periodic part of the PC field as well the PC dielectric constant in a Fourier series, the $z$ dependence of $P_{i j}$ and $K_{i j}$ can be written in terms of superpositions of $\exp \left[i\left(\beta_{i}-\beta_{j}-2 \pi m / \Lambda_{z}\right) z\right]$ where $m$ is an integer. For weak coupling $\left(\mathrm{d} C_{j} / \mathrm{d} z \ll 1 / \lambda\right)$, only the slowly varying component (compared with $\lambda$ ) of $K_{i j}$ significantly couples the amplitude coefficients $C_{i}$ and $C_{j}$ over laboratory 
length scales of interest. This reasoning is analogous to (for example) that used in the time-domain rotating-wave approximation in quantum mechanics and is often used to derive approximate analytic solutions to coupled-mode equations describing two-mode electromagnetic systems in the presence of weak gratings. ${ }^{40}$ Because of the strong dielectric contrast of the $\mathrm{PC}$, the problem here is more complex; however, the fundamental results from the simple cases hold: To observe significant power exchange between modes, their wave numbers $\beta$ must differ by approximately a reciprocal lattice vector $2 \pi m / \Lambda_{z}$. Also, the larger the phase-matched driving terms, the stronger the coupling. The mixing of the Fourier components of the PC Bloch mode and the dielectric constant is the most significant effect captured by this coupled-mode theory compared with standard weak grating theories. Physically this allows coupling between PC and fiber modes that is mediated either directly by a Fourier coefficient of the PC Bloch mode (the dominant effect here) or indirectly by the $\mathrm{PC}$ dielectric acting like a grating (a higherorder effect). Optimizing the magnitude of these coefficients and, as a result, the coupling from the fiber mode to the desired PC waveguide mode is discussed in Section 3.

\section{3. $k$ SPACE DESIGN}

PC defect waveguides are formed when a line of defects is introduced into an otherwise two- or three-dimensionally periodic PC. Here we consider pseudo-2-D membrane structures whose typical geometry is shown in Fig. 1. In absence of the defects, the eigenmodes of the bulk 2-D slab are Bloch modes whose in-plane wave number $\mathbf{k}$ is a good mode label and that are bound to the slab if $\omega(\mathbf{k})$ is below the cladding and substrate light lines, i.e., $\omega(\mathbf{k})$ $<c k / n_{c, s}$ where $n_{s}$ and $n_{c}$ are the indices of refraction of the substrate and cladding, respectively (we do not consider bound modes that exist at special points in $\omega-\mathbf{k}$ space above the light line, as shown in Ref. 41). The airbridge membrane structures considered here have $n_{c}$ $=n_{s}=1$, maximizing the area in $\omega-\mathbf{k}$ space where bound modes exist and also ensuring that the bound modes of a fiber taper $\left(n_{f} \approx 1.45\right)$ do not leak into the PC substrate.

The PC modes can be classified as either even or odd depending on their parity under inversion about the $x-z$ mirror plane of the slab (see Fig. 1 for the coordinate system), and it can be shown that the lowest-order even modes (i.e., modes with no zeros in the $y$ direction) are TE-like whereas the lowest-order odd modes (i.e., modes with one zero in the $y$ direction) are TM-like. We only consider coupling to TE-like modes (the fiber can couple to either). Furthermore, we assume that the slab is thin enough to ensure that the frequencies of the second-order odd modes (which are also TE-like) are above the frequency range of interest, so only the fundamental TE-like mode needs to be considered. Figure 2 shows the approximate band structure of the fundamental TE-like modes of the bulk square-lattice PC slab considered in this paper. This band structure is calculated with an effective index 2-D plane-wave expansion model that takes into account the finite thickness of the slab but neglects the vector nature of the field, providing a useful guide for analysis of the $\omega$-k space properties of potential PC waveguide modes.

When a line defect is introduced, the discrete translational symmetry of the PC is reduced from two to one dimension, and consequently only the component $\beta$ of $\mathbf{k}$ parallel to the line defect remains a good mode label. The corresponding Bloch eigenmodes must satisfy Eq. (3), and the resulting band structure is approximately obtained when the bulk PC band structure is projected onto the first Brillouin zone of the defect unit cell $\left[\omega_{1-\mathrm{D}}(\beta)\right.$ $=\omega_{2-\mathrm{D}}(\mathbf{k} \mid \mathbf{k} \cdot \hat{u}=\beta)$ where $\hat{u}$ is parallel to the line defect] as shown in Fig. 3 and by a discrete set of modes whose field is localized to the defect region. In $\mathbf{k}$ space the localized and delocalized modes are characterized by

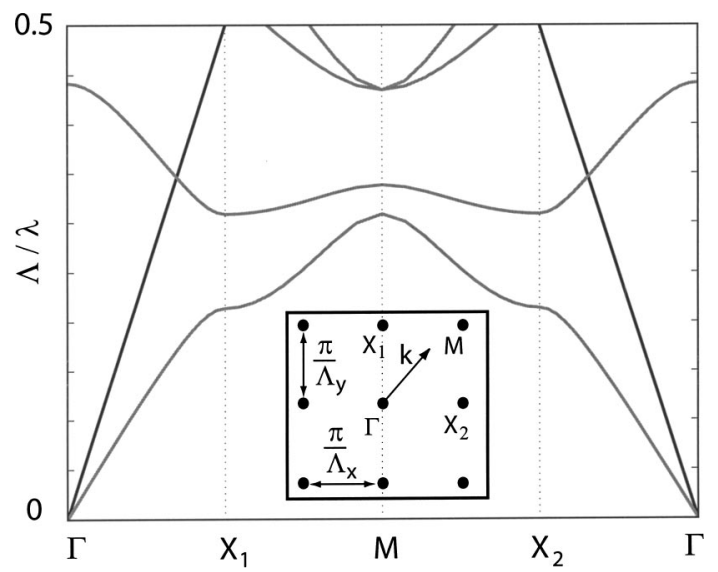

Fig. 2. Approximate band structure of fundamental even (TElike) modes for a square-lattice $\mathrm{PC}$ of air holes with radius $r / \Lambda$ $=0.35$ in a slab of thickness $d=0.75 \Lambda$ and dielectric constant $\epsilon=11.56$, calculated with an effective index of $n_{\mathrm{TE}}{ }^{\text {eff }}=2.64$ that corresponds to the propagation constant of the fundamental TE mode of the untextured slab. The inset shows the first Brillouin zone of a rectangular lattice.

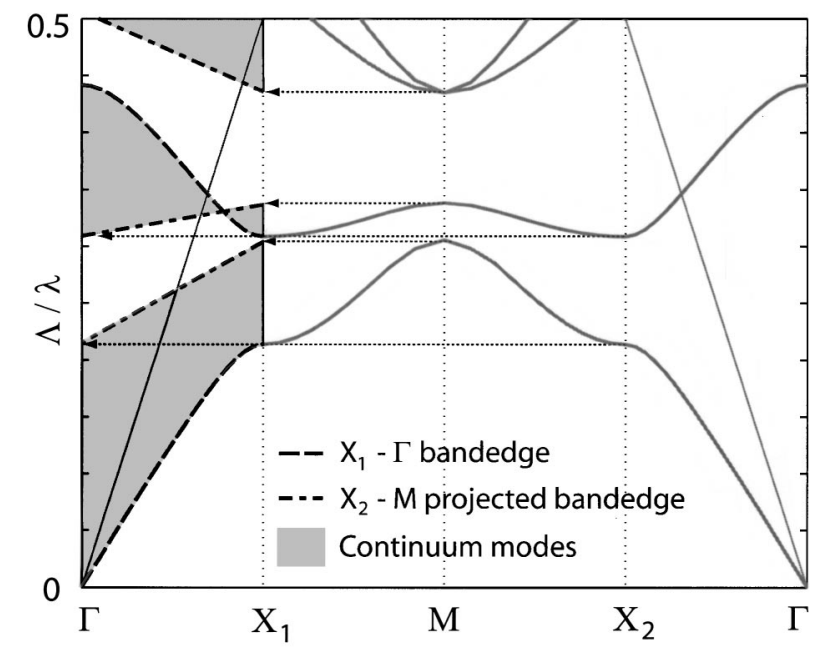

Fig. 3. Projection of the square-lattice band structure onto the first Brillouin zone of a line defect with the same periodicity of the lattice and oriented in the $X_{1} \rightarrow \Gamma$ direction. Band edges whose modes have dominant wave numbers in the $X_{1} \rightarrow \Gamma$ direction (i.e., $\mathbf{k}=k \hat{z}$ ) are shown by the dashed curves. Band edges whose modes have dominant wave numbers in the $X_{2} \rightarrow M$ direction (i.e., $\mathbf{k}=k_{z} \hat{z}+\pi / \Lambda_{x} \hat{x}$ ) are shown by the dot dashed curves. 
$\beta$ and a transverse wave-number distribution. The projection creates continuums of delocalized modes in $\omega-\beta$ space over which the dominant transverse wave number varies smoothly and approximates that of the bulk mode from which it is projected. For small defects the localized modes are superpositions of the delocalized modes at the top or bottom of the continuum regions, depending on whether the defect is an acceptor or donor type. By identifying from which bulk modes these continuum band edges are projected, we can thus approximately determine the dominant transverse wave number of the defect modes. Given a bulk 2-D band structure, we can therefore approximately determine the $\mathbf{k}$ space properties of the defect modes associated with any defect geometry without resorting to computationally expensive waveguide simulations.

To determine what PC waveguide $\mathbf{k}$ space properties are desirable for efficient coupling, it is necessary to consider the properties of the fiber taper mode. Guided fiber taper modes are confined to the region in $\omega-\beta$ space bounded by the air and fiber (usually silica, $n_{f} \approx 1.45$ ) light lines, as shown in Fig. 4, immediately limiting the PC modes that the fiber can phase match with. A suitable fiber typically has a radius of the order of a PC lattice constant, and the corresponding linearly polarized fundamental fiber mode $\left(\mathrm{HE}_{11} \pm \mathrm{HE}_{1-1}\right)$ is broad compared to the PC feature size. As a result, PC modes that are highly oscillatory in the transverse direction will not couple well to the fiber because their transverse coupling coefficients that were derived in Section 2 will be small. Thus PC modes that maximize the coupling coefficient must have a transverse wave-number distribution that is peaked at zero (i.e., have a large transverse dc component). This corresponds to defect modes that are dominantly formed from bulk PC modes whose $\mathbf{k}$ is parallel to the defect (the $X_{1} \rightarrow \Gamma$ direction here).

These ideas are illustrated in Fig. 4, which shows a bulk compressed square-lattice band structure projected onto the first Brillouin zone of a line defect in the $X_{1}$ $\rightarrow \Gamma$ direction. The compressed lattice is used for reasons discussed below and is not essential for the analysis. The approximate dispersion of localized modes formed by both donor- and acceptor-type defects are shown, and modes whose transverse wave-number distribution satisfy the requirements discussed above are indicated. It is not required that the defect modes be in a full PBG for the coupling scheme considered here. As in the case of other novel PC devices such as lasers ${ }^{42}$ and high- $Q$ cavities ${ }^{43}$ that have been realized in small bandgap square-lattice PCs, localized waveguide modes can exist without a full in-plane bandgap. ${ }^{44}$ That being said, for mode-selective coupling to be possible, it is necessary that the defect mode exists in a window in $\omega-\beta$ space, where the nearest mode degenerate in $\omega$ is detuned sufficiently in $\beta$ to suppress coupling due to its large phase mismatch. Although lattice compression is not required to achieve this, it is sometimes advantageous to distort the lattice to optimize the window in $\omega-\beta$ space, as was done here. Compressing the lattice in the transverse direction effectively raises the energy of the bands at the $X_{2}$ point and $M$ point in Fig. 3, modifying the projection of the full band struc-

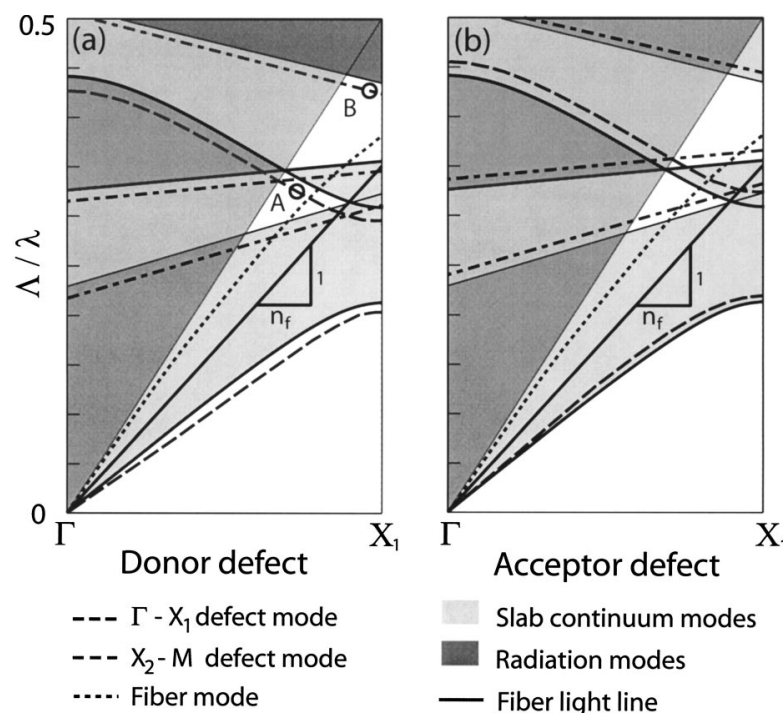

Fig. 4. Approximate projected band structure for a (a) donortype and (b) acceptor-type compressed square-lattice waveguide. Possible defect modes and the fundamental fiber taper mode are indicated by the dashed curves.

ture onto the first Brillouin zone of the defect waveguide, as reflected in Fig. 4.

Once an appropriate lattice and defect waveguide type have been selected and the approximate location of the desired mode in $\omega-\beta$ space is determined using these approximate 2-D techniques, we can use three-dimensional (3-D) FDTD to numerically calculate the field profiles and exact dispersion of the 3-D PC waveguide eigenmodes. The numerical results are used in turn in the coupledmode theory to model the coupling to the tapered fiber modes. These design principles are applied in Section 4 to design a compressed square-lattice PC defect waveguide that can couple efficiently to fiber tapers.

\section{CONTRADIRECTIONAL COUPLING IN A SQUARE LATTICE PHOTONIC CRYSTAL}

The PC waveguide modes considered in this paper are formed within an optically thin [thickness $t_{g}=(3 / 4) \Lambda_{x}$ ] semiconductor $(n=3.4)$ membrane perforated with a square array of air holes. From the approximate band structure for the bulk compressed square-lattice waveguide shown in Fig. 4, there are several potential defect waveguide modes that are not in a continuum and that can phase match with a fiber taper (whose typical dispersion is also shown). Of these modes, only waveguide mode $A$ has the desired transverse wave-number components: It comes off of a band edge projected from the $X_{1} \rightarrow \Gamma$ band of the bulk band structure, whereas the other modes come from $M \rightarrow X_{2}$ band edges. Because mode $A$ is not in a full-frequency bandgap, it is not an obvious candidate in the context of the existing literature that focuses on waveguide modes within a full bandgap. However, as we show, this mode is confined to the defect region, can be coupled selectively with a fiber taper, and can be used to probe high- $Q$ cavity modes.

A 3-D FDTD calculation of the even band structure for the graded waveguide of Fig. 1(c) in a compressed 
$\left(\Lambda_{x} / \Lambda_{z}=0.8\right)$ square lattice is shown in Fig. 5. Modes that are odd about the $x-z$ mirror plane are not shown in this plot; however, it was verified that the frequencies of the TE-like odd modes were higher than that of mode $A$ in the region of interest (circle A in Figs. 4 and 5). Although other donor defect geometries could have been used, the hole radius grading and the lattice compression used here are important design features of the waveguide for a number of reasons. As discussed in Section 6, the field profile of the graded cavity mode in Ref. 43 is similar to that of waveguide mode $A$, suggesting that this waveguide mode is ideal to tunnel light into and out of these cavities. In addition, the compressed lattice provides for (i) expansion of the window in $\omega-\beta$ space supporting defect donor-type modes that can phase match with the taper, (ii) an increase in the slope of the defect mode dispersion resulting in increased coupler bandwidth, and (iii) matching of the frequencies of the waveguide mode and the uncompressed defect cavity donor mode without any stitching of the lattice required (choosing $\Lambda_{x}{ }^{\mathrm{PC}}=\Lambda_{x}{ }^{\mathrm{Cav}}$ requires $\left.\Lambda_{z}{ }^{\mathrm{PC}} / \Lambda_{z}{ }^{\mathrm{Cav}}=\widetilde{\omega}^{\mathrm{Cav}} / \widetilde{\omega}^{\mathrm{PC}}\right)$. The two sets of localized states expected from Fig. 4 can be seen to form, one originating from the $X_{1}$ point in the 2-D reciprocal lattice and the other from the $M$ point. The most localized of each set are the fundamental (transverse) modes, which we label as mode $A$ and mode $B$ in Figs. 4 and 5. The magnetic field profiles and the transverse Fourier transforms of these localized modes are shown in Figs. 6 and 7. The Fourier transforms confirm that the dominant transverse Fourier components of mode $A$ are centered about $k_{x}=0$, whereas those of mode $B$ are centered about $k_{x}= \pm \pi / \Lambda_{x}$. Both of these modes have negative group velocity, indicating that coupling to them from the fiber will be contradirectional in nature.

Using the FDTD-calculated fields for the PC modes, the exactly calculated fields of a fiber taper, and including

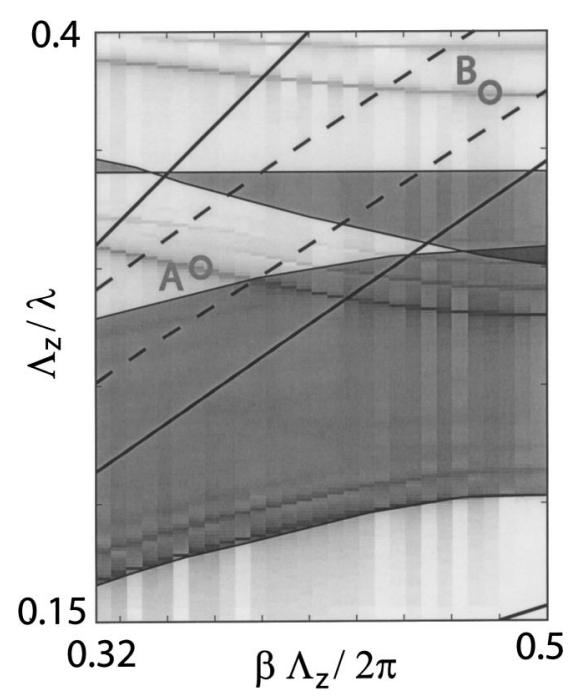

Fig. 5. 3-D FDTD calculated band structure for the waveguide shown in Fig. 1(c). The shaded regions indicate continuums of unbound modes. The dashed lines are the dispersion of fiber tapers with radius $r=0.8 \Lambda_{z}=1 \Lambda_{x}$ (upper dashed line) and $r$ $=1.5 \Lambda_{z}=1.875 \Lambda_{x}$ (lower dashed line). The solid lines are the air (upper line) and fiber (lower line) light lines. The energies and wave numbers of modes $A$ and $B$ are $\widetilde{\omega} \Lambda_{z} / 2 \pi$ $=0.304$ and 0.373 at $\beta \Lambda_{z} / 2 \pi=0.350$ and 0.438 , respectively.
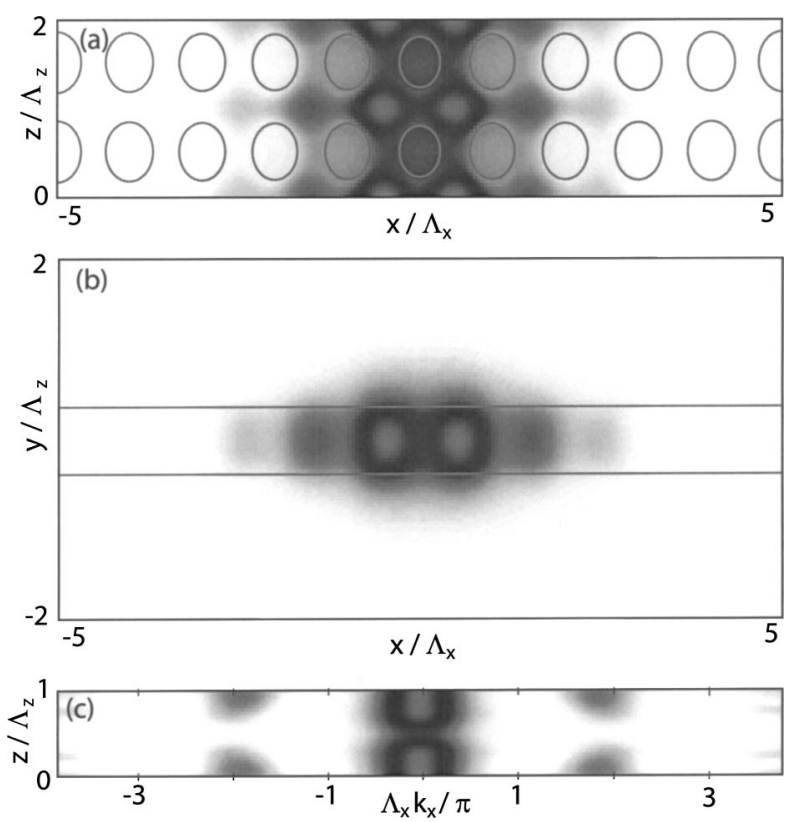

Fig. 6. Mode $A$ field profiles calculated by FDTD. Dominant magnetic field component (a) $\left|B_{y}(x, y=0, z)\right|$, (b) $\mid B_{y}(x, y$, $z=0) \mid$. (c) Dominant electric field component transverse Fourier transform $\left|\widetilde{E}_{x}\left(k_{x}, y=0, z\right)\right|$. Note that the dominant transverse Fourier components are near $k_{x}=0$.
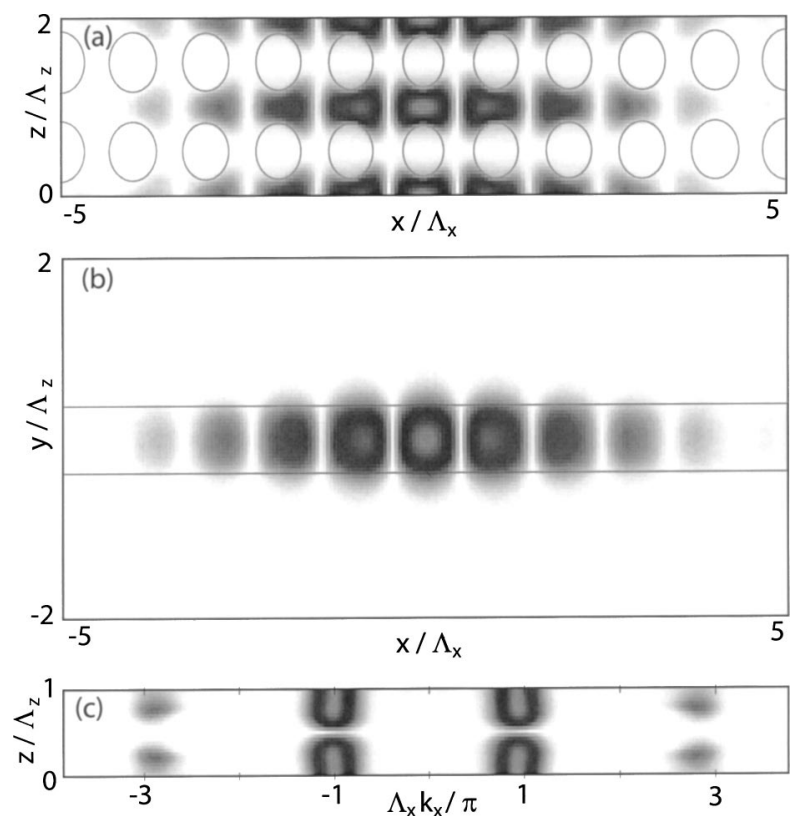

Fig. 7. Mode $B$ field profiles calculated by FDTD. Dominant magnetic field component (a) $\left|B_{y}(x, y=0, z)\right|$, (b) $\mid B_{y}(x, y$, $z=0) \mid$. (c) Dominant electric field component transverse Fourier transform $\left|\widetilde{E}_{x}\left(k_{x}, y=0, z\right)\right|$. Note that the dominant transverse Fourier components are near $k_{x}= \pm \pi / \Lambda_{x}$.

only those PC and fiber modes that are nearly phase matched (as well as their backward-propagating counterparts) in the coupled-mode theory, we calculated the mode amplitudes at the coupler outputs as a function of coupler length and detuning of $\omega$ from the phase-matching frequency $\omega_{0}$. Figure 8(a) shows the resulting coupling to mode $A$ from the fundamental mode of a taper with radius 
$r \approx 1.15 \Lambda_{x}$ placed at height $d=\Lambda_{x}$ above the PC. Figure 8 (b) shows the power in all four modes as a function of coupler length at the phase-matching condition. For reference, at an operating wavelength $\left(\lambda_{0}\right)$ of $1.55 \mu \mathrm{m}, \Lambda_{x}$ $\approx 0.5 \mu \mathrm{m}$, which corresponds to a taper diameter $(2 r)$ of roughly $1 \mu \mathrm{m}$ and a waveguide-to-waveguide gap $(d)$ of $0.5 \mu \mathrm{m}$ in this case. For $\omega=\omega_{0}$ and $L=50 \Lambda_{z}$, the coupled power is greater than $80 \%$ and reaches $95 \%$ for $L=80 \Lambda_{z}(\approx 40 \mu \mathrm{m})$. The remaining power is coupled to the backward-propagating fiber mode. Note that, because the PC mode has negative group velocity, this is contradirectional coupling resulting in monotonically increasing power transfer as a function of coupler length when the transverse coupling is stronger than the detuning in $\beta .^{45}$ The bandwidth is approximately $1.5 \%$ of $\omega_{0}$; we verified that, within this frequency range, coupling to other modes is negligible due to large phase mismatching. It should be noted that we could obtain shorter coupling lengths and larger coupling bandwidths by reducing the coupling gap $d$; however, in the model used here this would result in stronger coupling to the backwardpropagating fiber mode and a decreased asymptotic coupling efficiency. In addition, such strong coupling is best modeled with a more complete basis within coupled-mode theory or with a fully numerical approach such as FDTD.

To illustrate the importance of a mode's dominant transverse Fourier components for efficient coupling, Fig. 9 shows the power transfer as a function of coupler length and detuning to mode $B$ in Fig. 5 from an appropriately phase-matched fiber taper placed at $d=\Lambda_{x}$ above the
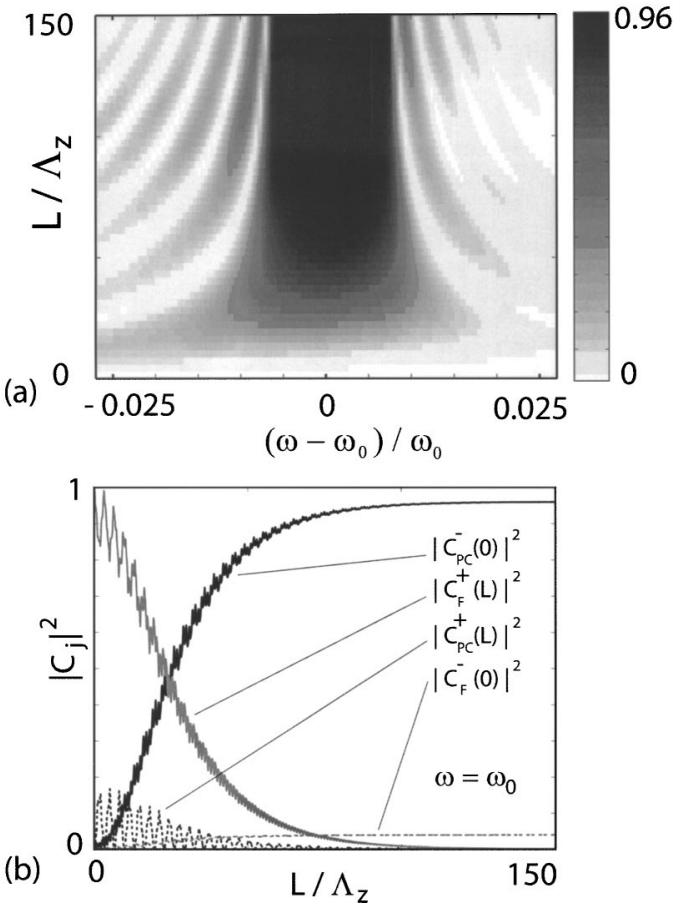

Fig. 8. (a) Power coupled to PC mode $A$ from a tapered fiber with radius $r=1.15 \Lambda_{x}$ placed with a $d=\Lambda_{x}$ gap above the PC as a function of detuning from phase matching and coupler length. (b) Power coupled at $\omega=\omega_{0}$ to the forward- and backward-propagating PC and fiber modes as a function of coupler length.

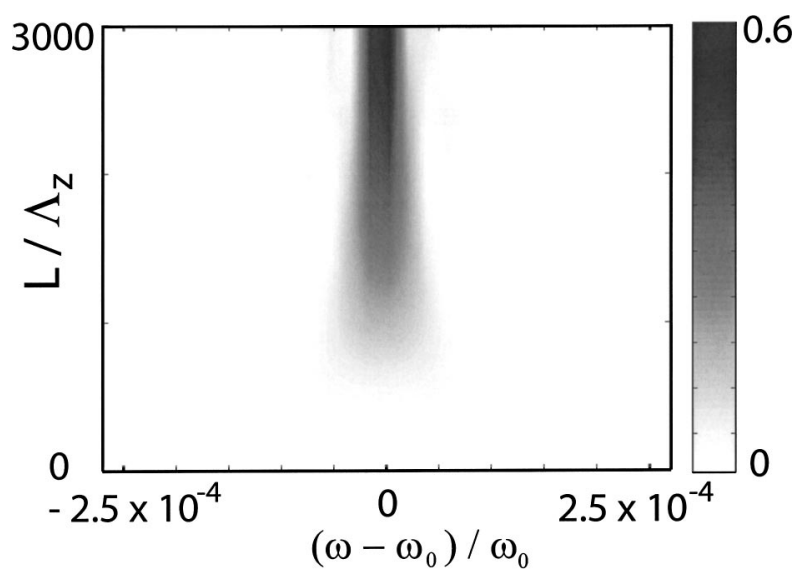

Fig. 9. Power coupled to PC mode $B$ from a tapered fiber with radius $r=1.55 \Lambda_{x}$ placed with a $d=\Lambda_{x}$ gap above the $\mathrm{PC}$ as a function of detuning from phase matching and coupler length.

PC. ${ }^{46}$ Although mode $B$ is even about the mirror plane in the center of the waveguide, because it is constructed from Bloch modes around the $M$ point it has relatively small amplitude for transverse Fourier components near zero, resulting in a small transverse overlap factor $\left(K_{i j}\right)$ with the fiber taper mode. This results in a coupler length $\approx 200$ times longer than that for mode $A$, as well as an extremely narrow bandwidth of $\approx 10^{-4} \%$ of $\omega_{0}$ (a property further amplified by mode $B$ 's low group velocity). Calculations not shown here from our studies of acceptor defect modes rising from the valence band edge $\left(M-X_{2}\right)$ yield similar results, despite their broad field profiles that would be expected to match well with the fiber.

These calculations demonstrate that, by selecting a mode composed from the appropriate regions in $\mathbf{k}$ space, we can achieve efficient power transfer between a tapered fiber and the PC that is mode selective and that (thanks to its contradirectional character) does not depend sensitively on the coupling length above some critical minimum. Using a more numerically intensive supermode calculation (and before we discuss the integration of this waveguide with high- $Q$ cavities), we now confirm that the simple coupling analysis used above is valid.

\section{SUPERMODE CALCULATIONS}

To verify the coupling between the individual waveguide modes presented in Section 4, it is useful to calculate the band structure of the hybrid fiber-PC waveguide system. Because this system retains the discrete translational symmetry of the PC waveguide, the band structure of its modes (the supermodes) can be calculated by FDTD with a combination of Bloch and absorbing boundary conditions in a similar manner as the band structure of the isolated PC waveguide. The resulting band structure provides information about the coupling between the modes of the individual waveguides. For weak coupling, it resembles the superposition of the individual waveguide band structures (for example, Fig. 5), but with anticrossings where the modes intersect and are coupled. The amount of deflection at an anticrossing is related to the 
strength of the coupling between the modes and can be used to back out physical parameters that describe the power transfer.

Figures 10(a) and 10(b) show the band structure for a fiber taper of radius $r=1.17 \Lambda_{z}=1.46 \Lambda_{x}$ placed at $d=\Lambda_{z}=1.25 \Lambda_{x}$ above the PC waveguide studied in Sections $2-4$. These parameters differ slightly from those used in Section 4, but they do not change the results significantly. The larger separation results in a longer coupling length and a smaller bandwidth, whereas the larger fiber radius lowers the phase-matching frequency slightly. The mirror symmetry about the $y-z$ plane of the fiber-PC system is used to filter for modes that are even about this plane; but the fiber breaks the mirror symmetry in the $x-z$ plane, and the band structure contains PC modes that are odd in the vertical direction and that are not shown in the band structures of Sections 3 and 4 . The dispersion of the individual fiber mode and the PC waveguide mode $A$ can be identified, and the anticrossing where they intersect indicates that the two modes are coupled. In addition, the fundamental fiber taper mode couples strongly to a series of PC modes at higher frequencies than mode $A$, which were not predicted from the analysis in Section 4. These are the above-mentioned second-order odd (about the $x-z$ plane) valence-band TElike PC modes. We can push these modes to higher frequencies faster than the fundamental TE-like modes by reducing the slab thickness (i.e., they can be frozen out of the frequency range of interest). However, their odd parity in the vertical direction results in an electric field amplitude maxima near the PC surface, and they interact strongly with the fiber taper. Figures 10(c) and 10(d)
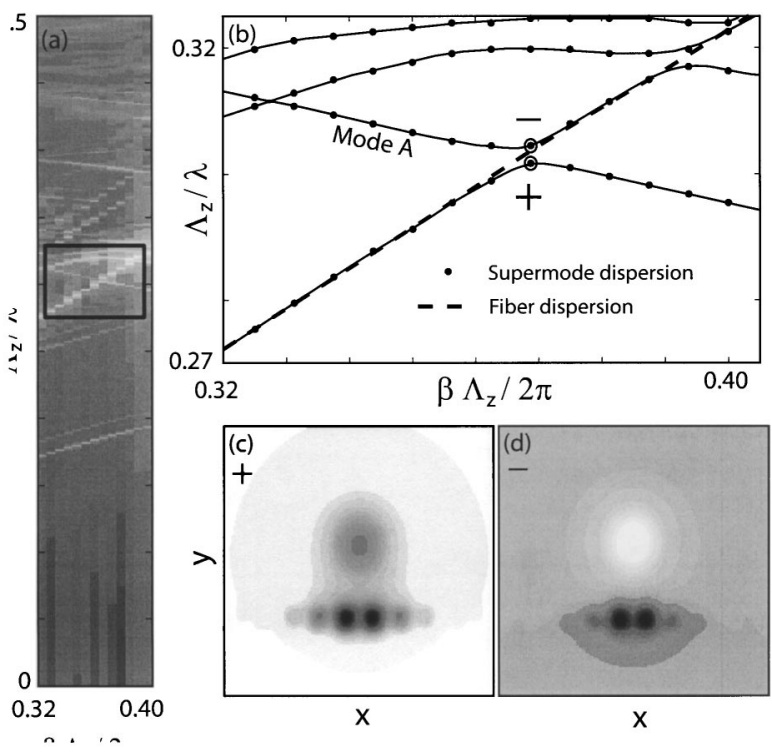

Fig. 10. (a) FDTD-calculated band structure of the full fiber-PC system. The fiber taper has a radius $r=1.17 \Lambda_{z}=1.46 \Lambda_{x}$ placed at $d=\Lambda_{z}=1.25 \Lambda_{x}$ above the PC waveguide. (b) Band structure of the boxed region in (a). Mode $A$ and fiberlike dispersion are identified, and the symmetric and antisymmetric superpositions of these modes at the anticrossing are labeled by the plus and minus signs. (c) The $B_{y}(x, y, 0)$ component of the lower-frequency anticrossing edge supermode (symmetric). (d) The $B_{y}(x, y, 0)$ component of the higher-frequency anticrossing edge supermode (antisymmetric). show the field profiles of the supermodes from either side of the anticrossing of interest. The low- and highfrequency supermodes closely resemble even and odd superpositions, respectively, of the individual waveguide modes, consistent with standard results for coupling between degenerate modes in (for example) guided-wave optics. $^{40}$

Because PC mode $A$ and the fiber mode have group velocities with opposite signs, a bandgap is formed where they anticross. This is consistent with the physical picture of the coupling: On resonance, a contradirectional coupler acts like a mirror and reflects the power from the forward-propagating mode into the backwardpropagating mode. Mathematically this is manifest in a nonzero imaginary part of the propagation constant $\beta$ inside the gap. Thus the supermodes propagate evanescently inside the gap, and complete contradirectional coupling is possible over a bandwidth equal to the size of the gap. The gap at the anticrossing of mode $A$ and the fiber mode in Fig. 10(b) has a width of $1 \%$ of its center frequency $\omega_{0}$. Coupled-mode calculations similar to those of Section 4 for a fiber radius and $\mathrm{PC}$-taper gap equal to those used in this section also yield a bandwidth of $1 \%$ of $\omega_{0}$.

\section{COUPLING TO HIGH-Q CAVITIES}

In Ref. 43, a group theoretical is used to design a cavity in a square-lattice PC slab that supports a high- $Q\left(\approx 10^{5}\right)$ defect mode. As alluded to above, the properties of this defect mode in part motivated the design of waveguide mode $A$, and in this section we demonstrate how the resultant similarities allow the cavity and waveguide to be simply and efficiently integrated.

We begin with a brief description of the cavity mode of Ref. 43. As the first-order bandgap energy minima of the conduction band in a square lattice occur at the $X_{1,2}$ points of the irreducible part of the Brillouin zone, donor modes from this band are expected to be composed of Fourier components in a neighborhood of the $\pm \mathbf{k}_{X_{1}}$ and $\pm \mathbf{k}_{X_{2}}$ points in $\mathbf{k}$ space (see Fig. 2). By considering the pointgroup symmetry of a given defect cavity and analyzing the allowed symmetries of modes it will support, it is possible to determine approximate forms of the localized defect cavity modes using group theory. Each of the allowed modes is labeled by an irreducible representation of the defect cavity's point group. Using $\mathbf{k}$ space arguments, the authors of Ref. 43 show that the mode that transforms as the $A_{2}$ representation of $C_{2 v}$ maximizes the cavity $Q$, and as such it is this mode that we consider here. In the horizontal mirror plane of the PC slab, the magnetic field of this mode has the approximate form

$$
\mathbf{B}=\hat{y}\left[\cos \left(\mathbf{k}_{X_{1}} \cdot \mathbf{r}_{\perp}\right)\right] \Gamma\left(\mathbf{r}_{\perp}\right),
$$

where $\mathbf{r}_{\perp}$ is the displacement from the center of the defect and $\Gamma\left(\mathbf{r}_{\perp}\right)$ is an envelope localized to the defect region (an optical Wannier theorem can be employed to calculate the envelope as shown in Ref. 47).

The results of FDTD simulations of the high- $Q$ defect mode of Ref. 43 are shown in Fig. 11. Its dominant 
k space components result in a highly directional mode profile along the $\hat{z}$ direction, parallel to the $\Gamma-X_{1}$ direction of the reciprocal lattice. The defect cavity supporting this defect mode consists of a square-lattice graded donor defect with $C_{2 v}$ symmetry. The hole radius in this cavity was graded approximately quadratically in both inplane directions [the $\hat{x}$ and $\hat{z}$ directions as depicted in Fig. 11(a)]. An identical grade as that in the $\hat{x}$ direction of the defect cavity was chosen for the lateral cross section of the waveguide studied here in anticipation of integration with this cavity. The resultant similarity in the lateral $(\hat{x}$-direction) mode profile of the waveguide and cavity modes is clear from the mode plots of Figs. 6(a) and 6(b) and Figs. 11(a) and 11(b), respectively. The lateral overlap factor of these two modes is $\left|\left\langle B_{y}{ }^{\mathrm{WG}} \mid B_{y}{ }^{\mathrm{Cav}}\right\rangle_{x y}\right| \sim 0.98$, where the waveguide and cavity fields are evaluated at their antinodes in the $\hat{z}$ direction.
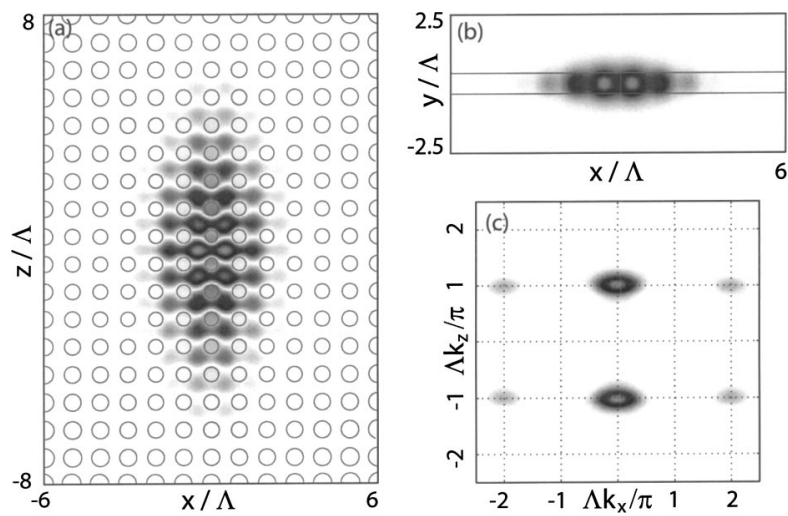

Fig. 11. High- $Q$ defect cavity mode of Ref. 43. Plots of the magnetic field pattern are shown in (a) the $x-z$ plane $\left[\mid B_{y}(x\right.$, $y=0, z) \mid]$ and (b) the $x-y$ plane $\left[\left|B_{y}(x, y, z=0)\right|\right]$. In (c) the Fourier transform of the dominant electric field component is plotted $\left[\left|\widetilde{E}_{x}\left(k_{x}, 0, k_{z}\right)\right|\right]$.

(a)

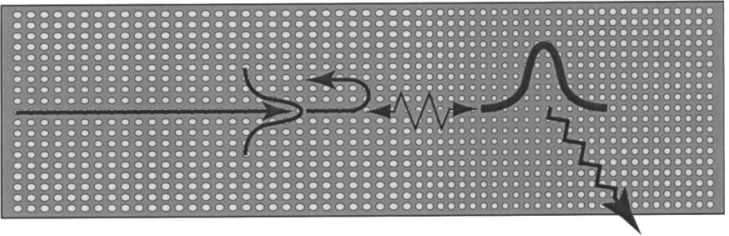

(b)

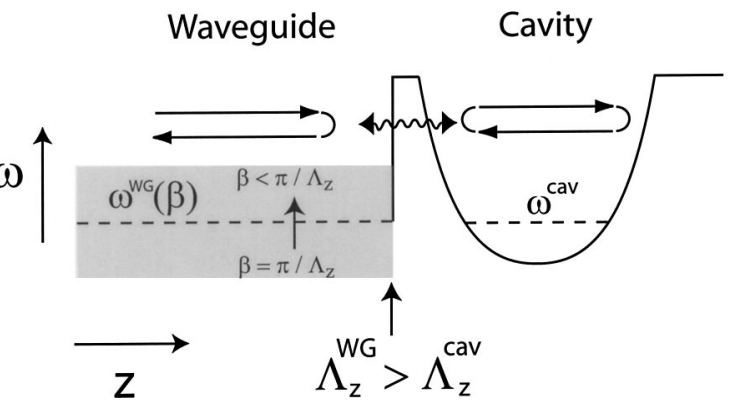

Fig. 12. (a) Waveguide to high- $Q$ cavity coupling scheme. (b) Schematic mode frequency diagram. The dotted line represents the cavity mode frequency. The solid black curve represents the local laterally guided-mode frequency and is a function of the filling fraction and lattice compression. The shaded region represents the finite bandwidth of the waveguide mode, which is a function of its dispersion (a flatter band has a smaller bandwidth and vice versa).
Figure 12(a) shows the proposed waveguide-cavity coupling scheme. The axis of the PC waveguide is aligned along the direction of the dominant Fourier components of the cavity mode of Ref. 43 ( $\hat{z}$ direction). A buffer region consisting of a variable number of periods of the square photonic lattice is placed between the end of the waveguide and the defect cavity. As illustrated schematically in Fig. 12(b), light tunnels between the cavity and the waveguide through the barrier. In general we can control the efficiency and strength of coupling between the cavity and the waveguide by (i) adjusting the compression, or the filling fraction of the waveguide lattice so that waveguide mode $A$ is resonant in frequency with the cavity mode, and (ii) by tailoring the waveguide defect so that the waveguide and cavity modes have similar transverse field profiles. The later condition is necessary so that, on reaching the waveguide-cavity interface, a propagating waveguide mode will preferentially tunnel into the cavity mode (as opposed to coupling to other PC slab modes or radiation modes and vice versa for the radiation from the cavity mode).

Because of the similarity between the lateral grade in the hole radius of the $\mathrm{PC}$ in the waveguide and the cavity sections, the coupled waveguide-cavity structure can be approximately viewed as a one-dimensional system along the $\hat{z}$ direction. In this ideal view, the lateral confinement in the cavity and waveguide PC sections is single mode. Only the mode of the PC waveguide-cavity structure that shares the lateral field profile of the cavity and waveguide modes discussed above is considered. This simplified (lateral) single-mode picture is schematically represented in Fig. 12(b), where the frequency of the (local) fundamental guided mode is plotted versus $z$ for a fixed lateral grading rate in the $\mathrm{PC}$ hole radius. We tune the frequency of the lateral guided mode at different positions in $z$ by adjusting the nominal hole radius (lattice filling fraction) or the lattice constant in the $\hat{z}$ direction (lattice compression or stretching). The mode's dispersion in the PC waveguide section results in a finitefrequency bandwidth and is represented in Fig. 12(b) by the shaded region. To avoid the stitching of different lattices, the lateral lattice constant is kept constant $\left(\Lambda_{x}^{\text {cav }}\right.$ $\left.=\Lambda_{x}{ }^{\mathrm{WG}}\right)$. In this way one can engineer band offsets to produce tunneling barriers and create localized resonant cavities along the $\hat{z}$ direction. To couple from the waveguide to the cavity we must bring the waveguide mode into resonance with the localized cavity mode. For the waveguide and cavity mode system considered here, we achieved this degeneracy by adjusting the waveguide lattice compression $\Lambda_{x}{ }^{\mathrm{WG}} / \Lambda_{z}{ }^{\mathrm{WG}}$. In addition, for the designs studied here there is no discontinuity in the hole size. As such, the strength of the coupling between the resonant cavity and the waveguide modes is determined by the band offset due to the lattice compression of the waveguide (height of the barrier) and the number of buffer periods between the waveguide and the cavity sections (width of the barrier).

The validity of the above picture is dependent on the similarity of the lateral mode profiles of the cavity and waveguide and the density of other parasitic modes that are resonant with the cavity and waveguide mode of interest. These parasitic modes can come from a number of 
sources: (i) radiation modes above the light cone of the PC slab, (ii) bulk PC slab modes that are not laterally confined, and (iii) other laterally confined PC slab modes. The unloaded cavity (in the absence of the waveguide) of Ref. 43 radiates vertically into the air cladding of the PC slab and predominantly in $\mathbf{k}_{M}$ in-plane directions. To effectively load the cavity with the waveguide, the cavity mode must radiate preferentially into the waveguide mode of interest (as compared with the radiation into the parasitic modes of the waveguide section), and the strength of this radiation into the waveguide must be comparable to the unloaded cavity radiation loss (critical coupling occurs when they are equal). Note that a single guided-mode PC waveguide (i.e., a waveguide mode in a full in-plane PBG) does not guarantee efficient waveguide-cavity coupling as the cavity mode may still radiate into the vertical radiation modes of the $\mathrm{PC}$ waveguide (as in the case of poorly matched cavity-waveguide lateral profiles).

We performed an analysis of the coupling between the waveguide designed here and the cavity of Ref. 43 using a 2-D effective index FDTD simulation of the full cavitywaveguide system. Although these 2-D simulations neglect vertical radiation loss, with this analysis we can determine how effectively the lateral profile of the waveguide mode has been matched to that of the cavity mode, a major consideration in our waveguide design and a necessity for efficient waveguide-cavity coupling as discussed above. Figure 13 shows the cavity mode in the presence of a series of waveguides with different lattice compressions. The magnetic field is shown at instants in time when it is a maximum (confined) and a minimum (radiating) in the cavity. Coupling to waveguide mode $A$ is negligible when the lattice is uncompressed because its lowest frequency (which occurs at the $X$ point) is higher than the cavity mode frequency. In this case the cavity radiates as if it were unloaded, and its dominant in-plane radiation is in the $\mathbf{k}_{M}$ directions. When the lattice is compressed, the waveguide mode frequencies are lowered, and the cavity becomes resonant with waveguide mode $A$

(a)

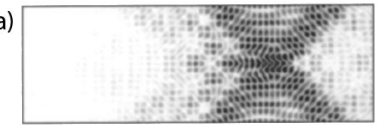

(b)

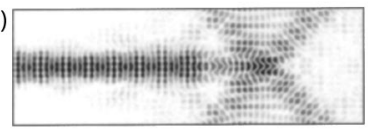

(c)

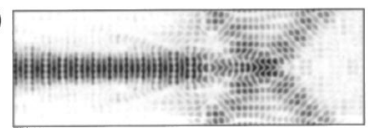

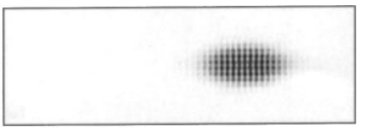
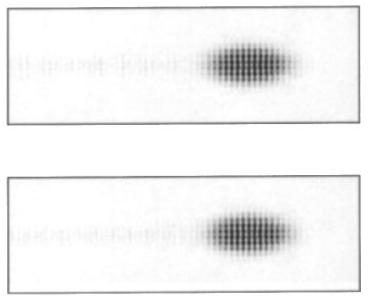

Fig. 13. Coupling from the defect cavity to the PC waveguide for varying waveguide lattice compressions at instances in time when the cavity magnetic field is a minimum (left) and a maximum (right). The envelope modulating the waveguide field is a standing wave caused by interference with reflections from the boundary of the computational domain. The diagonal radiation pattern of the cavity is due to coupling to the square-lattice $M$ points and is sufficiently small to ensure a cavity $Q$ of $\approx 10^{5}$. $|B|: \quad$ (a) $\Lambda_{x}{ }^{\mathrm{WG}} / \Lambda_{z}{ }^{\mathrm{WG}}=20 / 20$, (b) $\Lambda_{x}{ }^{\mathrm{WG}} / \Lambda_{z}{ }^{\mathrm{WG}}=20 / 25$ (ratio used in Section 4), (c) $\Lambda_{x}{ }^{\mathrm{WG}} / \Lambda_{z}{ }^{\mathrm{WG}}=20 / 29$. labeled by some propagation constant $\beta$ [see Fig. 12(b)]. The loaded cavity couples to mode $A$, but not into other (degenerate in $\omega$ but detuned in $\beta$ ) parasitic waveguide modes. As discussed above, this mode-selective coupling does not rely on a full PBG and is due to the similarity between the transverse profiles of the cavity and the waveguide modes of interest. The simulations also show that, because of the dispersion of mode $A$ and its corresponding frequency bandwidth, the waveguide-cavity coupling does not depend critically on the amount of lattice compression, so long as the compression is sufficient to lower mode $A$ 's frequency at the $X$ point below the cavity mode frequency. By tuning the lattice compression beyond this minimum, the propagation constant (and group velocity) of mode $A$ when it is resonant with the cavity mode is adjusted. Choosing the compression such that this propagation constant phase matches with a fiber taper $\left(\Lambda_{x} / \Lambda_{z}=20 / 25\right.$ for the case considered in the previous section), an efficient fiber taper-PC waveguide-PC cavity probe is realized.

\section{CONCLUSION}

Evanescent coupling from fiber tapers to defect waveguides in membrane PC structures has been studied by a general $\mathbf{k}$ space analysis, coupled-mode theory, and FDTD. A waveguide has been designed in a squarelattice PC that can be efficiently and simply coupled with a fiber taper and a high- $Q$ PC defect cavity, providing a simple optical probe for related PC devices. Contradirectional fiber-PC power transfer of $95 \%$ after 80 lattice constants $(\approx 50 \mu \mathrm{m})$ is expected, and it is shown that, when an appropriate waveguide lattice compression is chosen, the waveguide can be preferentially coupled with a high- $Q$ PC cavity whose mode has similar real and $\mathbf{k}$ space properties as the waveguide mode. These results, combined with the simplicity of the coupling scheme, suggest that evanescent coupling from fiber tapers will be an effective way of interfacing to PC devices.

\section{ACKNOWLEDGMENT}

K. Srinivasan thanks the Hertz foundation for financial support.

The e-mail address for P. E. Barclay is pbarclay @caltech.edu.

\section{REFERENCES AND NOTES}

1. E. Yablonovitch, "Inhibited spontaneous emission in solidstate physics and electronics," Phys. Rev. Lett. 58, 20592062 (1987).

2. P. S. J. Russell, D. M. Atkin, and T. A. Birks, in Microcavities and Photonic Bandgaps: Physics and Application, J. Warrby and C. Weisbuch, eds. (Kluwer Academic, Dordrecht, The Netherlands, 1996), pp. 203-218.

3. D. M. Atkin, P. S. J. Russell, T. A. Birks, and P. J. Roberts, "Photonic band structure of guided Bloch modes in high index films fully etched through with periodic microstructure," J. Mod. Opt. 43, 1035-1053 (1996).

4. M. Kanskar, P. Paddon, V. Pacradoui, R. Morin, A. Busch, J. F. Young, S. R. Johnson, J. Mackenzie, and T. Tiedje, "Observation of leaky slab modes in an air-bridged semiconduc- 
tor waveguide with two-dimensional photonic lattice," Appl. Phys. Lett. 70, 1438-1440 (1997).

5. S. G. Johnson, S. Fan, P. R. Villeneuve, J. D. Joannopoulos, and L. A. Kolodziejaki, "Guided modes in photonic crystal slabs," Phys. Rev. B 60, 5751-5758 (1999).

6. S. G. Johnson, P. R. Villeneuve, S. Fan, and J. D. Joannopoulos, "Linear waveguides in photonic-crystal slabs," Phys. Rev. B 62, 8212-8222 (2000).

7. M. Lončar, D. Nedeljković, T. Doll, J. Vučković, A. Scherer and T. P. Pearsall, "Waveguiding in planar photonic crystals," Appl. Phys. Lett. 77, 1937-1939 (2000).

8. O. Painter, R. K. Lee, A. Yariv, A. Scherer, J. D. O’Brien, P D. Dapkus, and I. Kim, "Two-dimensional photonic bandgap defect mode laser," Science 284, 1819-1824 (1999).

9. A. Yariv, Y. Xu, R. K. Lee, and A. Scherer, "Coupledresonator optical waveguide: a proposal and analysis," Opt. Lett. 24, 711-713 (1999).

10. N. Stefanou and A. Modinos, "Impurity bands in photonic insulators," Phys. Rev. B 57, 12127-12133 (1998).

11. S. Olivier, C. Smith, M. Rattier, H. Benisty, C. Weisbuch, T. Krauss, R. Houdré, and U. Oesterlé, "Miniband transmission in a photonic crystal coupled-resonator optical waveguide," Opt. Lett. 26, 1019-1021 (2001).

12. S. Noda, A. Chutinan, and M. Imada, "Trapping and emission of photons by a single defect in a photonic bandgap structure," Nature (London) 407, 608-610 (2000).

13. S. Fan, P. R. Villeneuve, and J. D. Joannopoulos, "Theoretical analysis of channel drop tunneling processes," Phys. Rev. B 59, 15882-15892 (1999).

14. G. Khitrova, H. M. Gibbs, F. Jahnke, M. Kira, and S. W Koch, "Nonlinear optics and normal-mode-coupling in semiconductor microcavities," Rev. Mod. Phys. 71, 1591-1639 (1999).

15. S. John and K. Busch, "Quantum electrodynamics near a photonic band gap: photon bound states and dressed atoms," Phys. Rev. Lett. 64, 2418-2421 (1990).

16. J. Vučković, M. Lončar, H. Mabuchi, and A. Scherer, "Design of photonic crystal microcavites for cavity QED," Phys. Rev. E 65, 016608 (2002)

17. Y. Yamamoto and S. Machida, "Microcavity semiconductor laser with enhanced spontaneous emission," Phys. Rev. A 44, 657-668 (1991).

18. P. Michler, A. Kiraz, C. Becher, W. V. Schoenfeld, P. M. Petroff, L. Zhang, E. Hu, and A. I. Glu, "A quantum dot single-photon turnstile device," Science 290, 2282-2285 (2000).

19. E. Moreau, I. Robert, J. M. Gerard, I. Abram, L. Manin, and V. Thierry-Mieg, "Single-mode solid-state single photon source based on isolated quantum dots in pillar microcavities,” Appl. Phys. Lett. 79, 2865-2867 (2001).

20. Y. Xu, R. K. Lee, and A. Yariv, "Adiabatic coupling between conventional dielectric waveguides and waveguides with discrete translational symmetry," Opt. Lett. 25, 755-757 (2000).

21. A. Mekis and J. D. Joannopoulos, "Tapered couplers for efficient interfacing between dielectric and photonic crystal waveguides," J. Lightwave Technol. 19, 861-865 (2001)

22. S. G. Johnson, P. Bienstman, M. A. Skorobogatiy, M. Ibanescu, E. Lidorikis, and J. D. Joannopoulos, "The adiabatic theorem and a continuous coupled-mode theory for efficient taper transitions in photonic crystals," Phys. Rev. E 66, 066608 (2002).

23. O. Mitomi, K. Kasaya, and H. Miyazawa, "Design of a single-mode tapered waveguide for low-loss chip-to-fiber coupling," IEEE J. Quantum Electron. 30, 1787-1793 (1994).

24. D. Taillaert, W. Bogaerts, P. Bienstman, T. F. Krauss, P. V. Daele, I. Moerman, S. Vertuyft, K. D. Mesel, and R. Baets,
"An out-of-plane grating coupler for efficient butt-coupling between compact planar waveguides and single-mode fibers," IEEE J. Quantum Electron. 38, 949-955 (2002).

25. T. A. Birks and Y. W. Li, "The shape of fiber tapers," J. Lightwave Technol. 10, 432-438 (1992).

26. W. Kuang, C. Kim, A. Stapleton, and J. O'Brien, "Gratingassisted coupling of optical fibers and photonic crystal waveguides," Opt. Lett. 27, 1604-1606 (2002).

27. P. E. Barclay, K. Srinivasan, M. Borselli, and O. Painter, "Experimental demonstration of evanescent coupling from optical fibre tapers to photonic crystal waveguides," IEE Electron. Lett. 39, 842-844 (2003).

28. M. Cai, O. Painter, and K. Vahala, "Observation of critical coupling in a fiber taper to a silica-microsphere whisperinggallery mode system," Phys. Rev. Lett. 85, 74-77 (2000).

29. D. K. Armani, T. J. Kippenberg, S. M. Spillane, and K. J. Vahala, "Ultra-high-Q toroid microcavity on a chip," Nature (London) 421, 925-928 (2003).

30. J. C. Slater, Microwave Electronics (Dover, New York, 1969).

31. W.-P. Huang, "Coupled-mode theory for optical waveguides: an overview,” J. Opt. Soc. Am. A 11, 963-983 (1994).

32. C. M. de Sterke, D. G. Salinas, and J. E. Sipe, "Coupledmode theory for light propagation through deep nonlinear gratings," Phys. Rev. E 54, 1969-1989 (1996).

33. N. A. R. Bhat and J. E. Sipe, "Optical pulse propagation in nonlinear photonic crystals," Phys. Rev. E 64, 056604 (2001).

34. S. Mookherjea and A. Yariv, "Second-harmonic generation with pulses in a coupled-resonator optical waveguide," Phys. Rev. E 65, 026607 (2002).

35. K. Sakoda, Optical Properties of Photonic Crystals (Springer, New York, 2001), pp. 14-19.

36. A. A. Barybin and V. A. Dmitriev, Modern Electrodynamics and Coupled-Mode Theory (Rinton, Princeton, N.J., 2002).

37. H. A. Haus, W. P. Huang, and A. W. Snyder, "Coupled-mode formulations," Opt. Lett. 14, 1222-1224 (1989).

38. A. W. Snyder and J. D. Love, Optical Waveguide Theory (Chapman \& Hall, New York, 1983).

39. W. P. Huang and H. A. Haus, "Power exchange in gratingassisted couplers," J. Lightwave Technol. 7, 920-924 (1989).

40. A. Yariv, Optical Electronics, 4th ed. (Saunders, Orlando, Fla., 1991).

41. P. Paddon and J. F. Young, "Two-dimensional vectorcoupled-mode theory for textured planar waveguides," Phys. Rev. B 61, 2090-2101 (2000)

42. H.-Y. Ryu, S.-H. Kim, H.-G. Park, J.-K. Hwang, Y.-H. Lee and J.-S. Kim, "Square-lattice photonic band-gap single-cell laser operating in the lowest-order whispering gallery mode," Appl. Phys. Lett. 80, 3883-3885 (2002).

43. K. Srinivasan and O. Painter, "Momentum space design of high-Q photonic crystal nanocavities in two-dimensional slab waveguides," Opt. Exp. 10, 670-684 (2002), http:// www.opticsexpress.org.

44. S. Kawakami, "Analytically solvable model of photonic crystal structures and novel phenomena," J. Lightwave Technol. 20, 1644-1650 (2002).

45. P. Yeh and H. F. Taylor, "Contradirectional frequencyselectice couplers for guided-wave optics," Appl. Opt. 19, 2848-2855 (1980).

46. In addition to the weak coupling between this mode and the fiber taper, higher-order odd slab modes may make coupling in this region of $\mathbf{k}$ space impractical. Nonetheless, the calculations shown here demonstrate the importance of a mode's transverse Fourier components.

47. O. Painter, K. Srinivasan, and P. E. Barclay, "Wannier-like equation for the resonant cavity modes of locally perturbed photonic crystals," Phys. Rev. B 68, 035214 (2003). 